\title{
Radio Astronomical Scintillation in the Solar Wind Plasma: Imaging Interplanetary Disturbances
}

\author{
P.K. Manoharan ${ }^{1}$, M. Pick ${ }^{2}$, and LASCO Consortium \\ ${ }^{1}$ Radio Astronomy Centre (NCRA), Tata Institute of Fundamental \\ Research, Ooty 643001, India \\ ${ }^{2}$ DASOP, Observatoire de Paris, 92195 Meudon, France
}

\begin{abstract}
When radio waves propagate through a irregular medium, scattering by the random refractive index inhomogeneities can lead to a wide variety of phenomena, which include intensity scintillation. The observed scattering can be interpreted to gain information about the random medium and such inversion studies are valuable when the accessibility of the medium becomes difficult. This paper briefly describes the intensity scintillation of celestial radio sources caused by the turbulence in the solar wind and summarizes the salient features of the method employed in mapping the structure of disturbances leaving the Sun out to $\sim 1$ AU.
\end{abstract}

\section{Interplanetary Scintillation}

The interplanetary scintillation (IPS) is observed when radiation from a compact radio source (size, $\theta<400$ mas) is scattered by the small-scale $(<500 \mathrm{~km})$ density irregularities in the solar wind. The degree of scintillation is given by the scintillation index, which is defined as the root mean square (rms) of radio intensity fluctuations $(i . e$. , scintillating flux density, $\delta \mathrm{S}$ ) normalized by the mean flux density of the source. Since the heliocentric distance, $R$, to the line of sight of a radio source changes due to the orbital motion of the Earth, the IPS measurements of a given source over many days provide the level of $\delta \mathrm{S}$ produced by the solar wind at various distances as well as at different position angles around the Sun (cf., Manoharan 1993). Figure 1 shows $\delta$ S of a radio source observed at various distances from the Sun, using the Ooty Radio Telescope(ORT) operating at $327 \mathrm{MHz}$ (Swarup et al. 1971). When the scattering is weak (at $\mathrm{R}>40$ $\mathrm{R}_{\odot} ; 1$ solar radius, $\left.\mathrm{R}_{\odot}=6.96 \times 10^{5} \mathrm{~km}\right)$, the intensity fluctuations are directly related to the rms of density fluctuations $\left(\delta \mathrm{N}_{e}\right)$ in the solar wind, i.e., $\delta \mathrm{S} \propto \delta \mathrm{N}_{e}$ (Manoharan 1993).

In Figure 1, one can notice large day to day variations of $\delta \mathrm{S}$ imposed upon a systematic decrease as the line of sight moves away from the Sun. The systematic decrease is due to the inverse $\mathrm{R}^{2}$ fall of $\delta \mathrm{N}_{e}$ (Manoharan 1993). However, when the line of sight to the radio source passes through an interplanetary disturbance (IPD), the scintillation shoots above the average level. Therefore, the systematic trend in the $\delta \mathrm{S}-\mathrm{R}$ curve can be removed by normalizing the observed scintillation with the expected average value and the normalized scintillation indices, $g \approx 1$, 


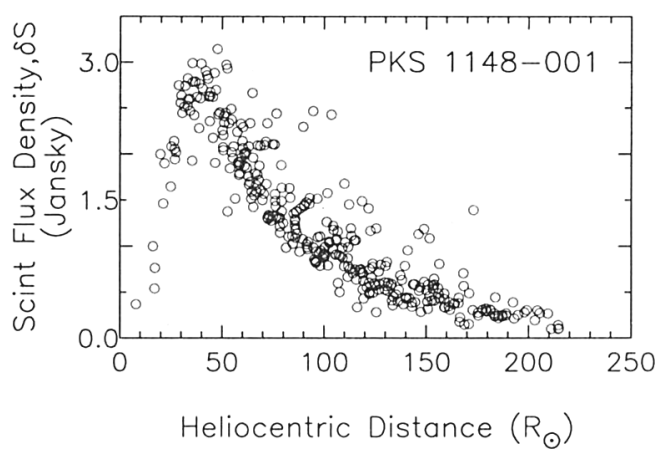

Figure 1. Scintillating flux density of the radio source, PKS1148-001, as a function of distance, $R$, from the Sun. It may be noted that the compact component size, $\theta$, of this source is $\sim 15$ mas and the measured scintillation is due to the contribution of the total flux density of the source, which is about $3.2 \mathrm{Jy}$ at $327 \mathrm{MHz}$.

$>1$ and $<1$, indicate the ambient level of solar wind, enhanced and depleted density fluctuations, respectively (Manoharan et al. 2000). Another interesting point is the possibility of estimation of solar wind speed by suitably calibrating the scintillation spectrum, which is derived from the intensity time series of each radio source (Manoharan \& Ananthakrishnan 1990).

\section{Imaging Interplanetary Disturbances}

When a grid of radio sources occupying different positions in IP space is employed, IPS method provides good spatial coverage. The source grid can also be advanced in space to achieve temporal sequence of a moving IPD. Such regular monitoring of inner IP space $\left(R \approx 50-200 R_{\odot}\right)$ made with the Ooty Radio Telescope, by observing $\sim 200$ to 300 radio sources in a day, has detected many interesting IPDs produced by coronal mass ejections (CMEs). Figure 2 shows an example of the sequence of propagation of a CME occurred on June 2, 1999 at 20:40 UT. The upper row shows the CME as observed by the LASCO - C2 and C3 coronagraphs on board SOHO spacecraft (Brueckner et al. 1995). The IPS images of density turbulence ( $g$-maps) obtained from the Ooty observations on 4 - 6 June 1999 are shown in the bottom row. These $g$-maps show the outward propagation of IPD associated with the CME and its evolution with distance.

Ooty images although show multiple CMEs moving in different directions, in particular, the arrival times of the western-side CME (in continuation with the LASCO measurements) at the window of IPS sources at different increasing distances provide the speed of the CME in the region between Sun and 1 $\mathrm{AU}\left(\simeq 215 \mathrm{R}_{\odot}\right)$. Figure 3 shows the speed-distance, $\mathrm{V}-\mathrm{R}$, plot of the CME obtained from the LASCO (C2 and C3) and IPS data. The speed estimates from the onset of CME at IPS window are in good agreement with the speed derived 


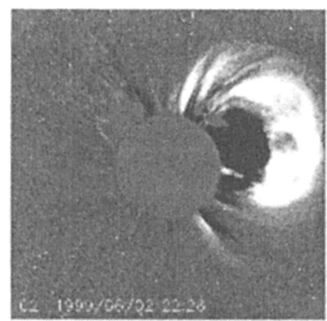

Ooty Scint. Doto 04 June 1999

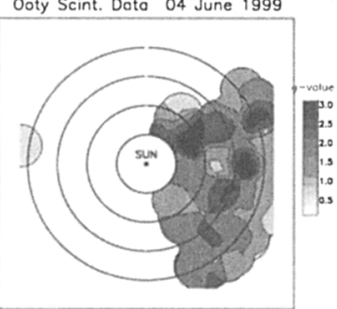

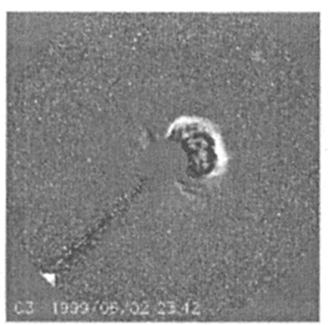

Ooty Scint. Doto 05 June 1999
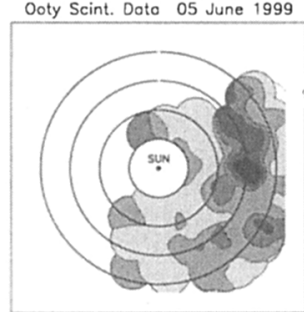

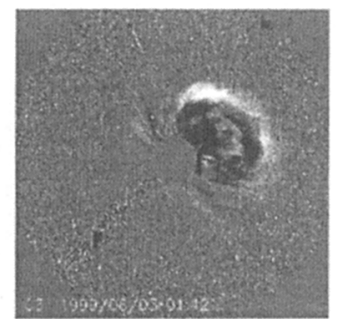

Ooty Scint. Doto 06 June 1999

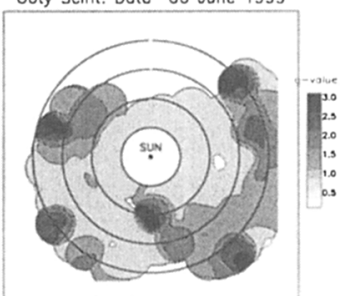

Figure 2. Partial halo CME of 02 June 1999 seen by LASCO - C2 and C3 coronagraphs (upper row) and Ooty scintillation images for 4 6 June 1999 (bottom row). In the upper row, first (2 June, 22:26 UT) and rest two images (2 June, 23:42 UT; 3 June, 01:42 UT) show C2 and C3 fields of view, respectively, which extend up to $\sim 6$ and $\sim 30 \mathrm{R}_{\odot}$. The concentric circles on the Ooty images are of radii 50,100,150, and $200 \mathrm{R}_{\odot}$. In these 'position angle - heliocentric distance' plots, Sun is at the center and top and right side of the plot represent, respectively, north and west of the Sun. On Ooty plots, the shade changes from light to dark for low to high $g$-values.

from the scintillation spectra of high $g$-values (Manoharan \& Ananthakrishnan 1990). It is evident from the log-scale plot (right-side plot) that in the near-Sun region, the deceleration of CME speed is marginal, $\mathrm{V}_{C M E} \sim \mathrm{R}^{-0.05}$ and where as at distances, $\mathrm{R}>80 \mathrm{R}_{\odot}$, it follows the relation, $\mathrm{V}_{C M E} \sim \mathrm{R}^{-0.35}$. A large body of IPS data of CMEs shows that the power-law index can vary between 0 to -1 , i.e., steadily moving CME to heavily decelerating CME with distance. It is likely that the power-law index depends on the initial speed of the CME (Manoharan et al. 2000).

\section{Summary}

The IPS and LASCO data presented here demonstrate that the IPS technique is an effective tool to detect propagating IPDs and it also provides otherwise unavailable information on size, speed and density turbulence of propagating CMEs in 3-D IP space. Such studies will also be useful in improving the predictive capabilities of intensity of magnetic storms at the near-Earth environment. Manoharan et al.(2000) have recently also shown that the density-turbulence 

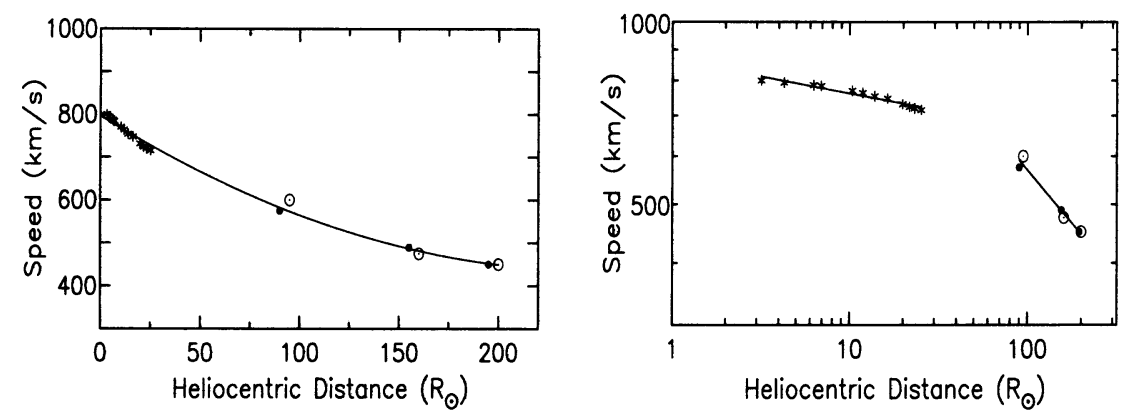

Figure 3. CME speed as a function of distance, R, from the Sun, plotted on linear (left) and log (right) scales. Symbols used are: 'star' speed derived from LASCO data, 'open circle' speed from onset time of disturbance at IPS source windows, and filled circle average speed from high $g$-values spectra (Manoharan \& Ananthakrishnan 1990). The distance dependence of speed is evident from the log-scale plot.

spectrum associated with the moving disturbance is significantly different from that of high-speed flow from coronal holes and low-speed wind originating above closed-field coronal streamers. A detailed study using a large number of IPS events is being carried out to understand the solar eruption and its manifestations in IP space.

Acknowledgments. We are grateful to the observing staff of the Radio Astronomy Centre, Ooty, for help with the observations. SOHO is an international project of cooperation between ESA and NASA. Part of this work is supported by CEFIPRA.

\section{References}

Brueckner, G.E., et al., 1995, Solar Phys., 162, 357.

Manoharan, P.K., 1993, 1993, Solar Phys., 148, 153.

Manoharan, P.K., \& Ananthakrishnan, S., 1990, MNRAS, 244, 691.

Manoharan, P.K., et al., 2000, ApJ, 530, 1061.

Swarup, G., et al., 1971, Nature Phys. Sci., 230, 185. 